\title{
An Overview of the Efficacy and Safety of Ozanimod for the Treatment of Relapsing Multiple Sclerosis
}

\author{
Marzia Fronza' \\ Lorena Lorefice ${ }^{2}$ \\ Jessica $\mathrm{Frau}^{2}$ \\ Eleonora Cocco (1D ${ }^{1,2}$ \\ 'Department of Medical Science and \\ Public Health, University of Cagliari, \\ Cagliari, Italy; ${ }^{2}$ Multiple Sclerosis Center, \\ Binaghi Hospital, ATS Sardegna, ASSL \\ Cagliari, Cagliari, Italy
}

\begin{abstract}
Multiple sclerosis (MS) is a complex disease of the central nervous system that can cause permanent disability in young adults. A large armamentarium is available for its management and is increasing over time. Ozanimod is an oral drug belonging to the sphingosine-1-phosphate receptor (S1PR) modulator family recently approved in different countries for MS with active disease. It selectively modulates S1PR1 and S1PR5 to prevent autoreactive lymphocytes from entering the central nervous system (CNS), where they can determine inflammation and neurodegeneration. Ozanimod was tested in one Phase II and two Phase III pivotal trials and was shown to be effective and well tolerated. Moreover, further investigations, including comparative trials with other S1P modulators and MS disease-modifying drugs, are needed to better define placement in MS treatment. Furthermore, ozanimod is currently under evaluation for inflammatory bowel diseases, such as ulcerative colitis and Crohn's disease, in international phase III studies. This article retraces the itinerary leading to the approval of ozanimod for MS treatment and its peculiarities and potentiality inside the S1PR modulator family.
\end{abstract}

Keywords: S1PR modulators, disease modifying therapy, brain atrophy, safety

\section{Introduction}

Multiple sclerosis (MS) is the most common inflammatory immune-mediated demyelinating disease of the central nervous system (CNS), affecting approximately 2.3 million people worldwide. ${ }^{1}$ It is one of the most common causes of disability in young adults. ${ }^{2}$ Although MS remains a chronic condition, early diagnosis and treatment are the keys to achieving satisfactory control of the disease. Currently, many disease-modifying drugs (DMDs) with different immunomodulatory mechanisms have become available and can radically change the natural progression of MS. Most of them have a significant effect on inflammation and are approved to treat relapsing-remitting forms of MS (RRMS); however, the longterm neuroprotective effects remain unproven.

\section{SIPR Modulators}

Correspondence: Eleonora Cocco Multiple Sclerosis Center, Binaghi Hospital, ATS Sardegna, ASSL, Via is Guadazzonis 2, Cagliari, 09126, Italy Tel +390706092806

Email ecocco@unica.it
Sphingosine 1-phosphate receptor (S1PR) modulators represent a class of oral drugs with a unique mechanism of action. These drugs are small molecule analogues of sphingosine 1-phosphate, a pleiotropic lipid mediator derived from ceramide, which is involved in several cellular functions, such as proliferation, 
migration, rearrangement of cytoskeleton, adhesion, and inflammation. ${ }^{3}$ By interacting with S1PR, the S1P analogue evokes several effects in different tissues and organs. $\mathrm{S} 1 \mathrm{PR}$ is a $\mathrm{G}$ protein-coupled receptor with five subtypes (S1PR1-5) that are expressed in multiple organs and systems. ${ }^{4}$ The primary therapeutic action of S1PR modulators is due to the inhibition of S1PR subtype 1 located on lymphocytes, neural cells, endothelial cells, smooth muscle cells, atrial myocytes, and atrioventricular (AV) nodes.5 Its inhibition blocks lymphocyte egress from lymph nodes and the thymus, resulting in a reduction of circulating lymphocytes and thus inflammatory cell infiltration in the CNS. Fingolimod (also called FTY720 and Gilenya $^{\mathrm{TM}}$ ), a novel immunosuppressant, induces the sequestration of circulating mature lymphocytes by accelerating lymphocyte homing in rats. An increase in the frequency of CD62L-positive T cells in Peyer's patches by fingolimod-induced lymphocyte homing was observed by Yanagawa. ${ }^{6}$

S1PR modulators can readily cross the blood-brain barrier (BBB), thereby allowing the establishment of a direct interaction with CNS receptors; this interaction may be partly responsible for the therapeutic benefit demonstrated by this class of drugs. ${ }^{7}$

The modulation of S1PR in the neurons, microglia, astrocytes, and oligodendrocytes has been hypothesized to promote myelin regeneration and to prevent the occurrence of synaptic defects, ultimately preventing neuronal damage. ${ }^{8}$ These effects are thought to be attributable not only to the interaction with the subtype 1 receptor, but also with the subtype 5 receptor that is mainly expressed on oligodendrocytes in white matter tracts and on brain endothelial cells. ${ }^{9,10}$ Interactions with S1PR5 may mediate oligodendrocyte survival that is essential for neuronal remyelination. ${ }^{9}$ It is also thought that S1PR5 expressed on CNS endothelial cells plays an important role in BBB formation and permeability maintenance. ${ }^{11}$

\section{Fingolimod}

The first oral drug developed and approved for the treatment of relapsing-remitting MS in 2010 was fingolimod, a small lipophilic agent that is phosphorylated by sphingosine kinase to become an S1P analogue. It binds to S1PR and induces its internalization; after fingolimod phosphate dissociation, the receptor returns to the surface. ${ }^{12}$ Fingolimod is a non-selective modulator of all S1PR subtypes, except for subtype 2. Clinical trials showed a $60 \%$ decrease in the annualized relapse rate (ARR) in fingolimod $1.25 \mathrm{mg} / \mathrm{d}$-treated patients versus placebo and a reduction in new $\mathrm{T} 2$ lesions and gadolinium-enhancing (GdE) lesions on MRI scans over 24 months of treatment. ${ }^{8}$ The good efficacy, tolerability, and convenient route of administration have been key to the success of fingolimod. Nevertheless, its non-selectivity leads to interactions with S1PR 3-4-5, which are likely responsible for several of fingolimod's adverse events, including QT interval prolongation, hypertension, macular oedema, pulmonary toxicity, and possibly hepatotoxicity. ${ }^{13}$ Moreover, the effects of fingolimod are reversible but are affected by its prolonged half-life of $7 \mathrm{~d}$.

The commercial success of fingolimod has prompted the development of new-generation S1PR modulators with greater selectivity for S1PR1 to achieve good efficacy while reducing safety concerns.

Several molecules are under trials; some of them have completed phase III trials and have been approved by the principal regulatory authorities.

\section{Siponimod}

Siponimod (also known as BAF312 and Mayzent ${ }^{\mathrm{TM}}$ ) is a highly selective functional antagonist of S1PR1 and S1PR5, with very low activity exerted on the S1P2, $\mathrm{S} 1 \mathrm{P} 3$, and S1P4 receptors. Selectivity for S1P1 versus $\mathrm{S} 1 \mathrm{P} 2$, S1P3, and S1P4 receptors is reportedly greater than 1000 -fold. ${ }^{14}$ With a relatively short half-life of 30 hours, the once-daily oral dosing regimen is permitted; recovery of the absolute lymphocytes count (ALC) to baseline levels is observed in most subjects within 10 days after treatment discontinuation. ${ }^{7}$ A phase II clinical trial in RRMS (BOLD) ${ }^{15}$ and a subsequent extension study ${ }^{16}$ were completed and showed a reduction in new T2 lesions and GdE lesions at three months for siponimod versus placebo.

The phase III trial inactive secondary progressive MS (SPMS) EXPAND ${ }^{17}$ met its primary endpoint, as siponimod reduced three-month confirmed disability by $21 \%$ versus placebo. The results of these trials suggest a role of siponimod in the reduction of disease activity with a modest effect on the gradual disability accrual, ${ }^{18}$ with younger patients with more active forms of SPMS being the most likely to benefit. ${ }^{4}$

In light of this evidence, in March 2019, the Food and Drug Administration (FDA) ${ }^{19}$ approved siponimod for active forms of MS, including clinically isolated syndrome (CIS), RRMS, and active SPSM, while the European 
Medicines Agency (EMA) approved siponimod in January 2020 for active SPMS. ${ }^{20}$

\section{Ponesimod}

Ponesimod (ACT-128800, Ponvory ${ }^{\mathrm{TM}}$ ) is another novel S1PR modulator with a high target selectivity for S1PR1 and a short half-life. A phase IIb trial ${ }^{21}$ has shown that the cumulative number of new GdE lesions was reduced in the ponesimod group versus placebo. The OPTIMUM ${ }^{22}$ Phase III study has revealed a $30 \%$ reduction of the ARR in relapsing MS patients prescribed with ponesimod compared to patients prescribed with teriflunomide $(0.202$ vs 0.290). Ponesimod also showed superior results to teriflunomide in analyses involving magnetic resonance imaging activity, brain volume loss, fatigue, and showed no evidence of disease activity (NEDA) status. No differences in disability progression were noted in the two treatment groups. The safety profile of ponesimod was in line with that reported by previous trials, with $8.7 \%$ of the serious treatment-emergent adverse events (TEAEs) resulting in treatment discontinuation.

In March 2021, the Committee for Medicinal Products for Human Use (CHMP) of EMA expressed a positive opinion on ponesimod, recommending that a marketing authorisation should be granted for the medicinal product Ponvory for the treatment of active relapsing forms of multiple sclerosis. ${ }^{23}$

\section{Ozanimod}

Also known as RPC1063 and Zeposia ${ }^{\mathrm{TM}}$. The drug main specifics are shown in Table 1.

\section{Pharmacodynamics}

Ozanimod is an agonist of S1PR1 and S1PR5 with 27-fold selectivity for S1PR1 over S1PR5. Its interaction with S1PR leads to receptor internalization and subsequent ubiquitin-proteasome-dependent degradation, ${ }^{24}$ preventing receptor reinstallation in the cellular membrane. This leads to the inhibition of lymphocyte egress from lymph nodes, causing a decrease in the absolute lymphocyte count (ALC). The reduction of ALC is dose-dependent, with a plateau effect at a dose of $1 \mathrm{mg} / \mathrm{d}$, and displays a preference for lymphocyte subtypes, with increased effects on CD4+ CCR7+ and CD8+ CCR7+ T cells. ${ }^{25}$ This lymphocyte subset selectivity has also been shown for other S1P modulators, suggesting a class ability to maintain protective immunity while targeting the immunopathologic pathway of MS. ${ }^{26}$ Earlier studies showed that the effect of ozanimod was rapidly reversible, with ALC restored within 48-72 hours after drug discontinuation. ${ }^{25}$ However, recently studies reported a longer time to ALC recovery, ie, up to 30 days after ozanimod withdrawal. ${ }^{7}$ This is mainly attributable to the long half-life of CC112273, ozanimod's major active metabolite. The slow return of lymphocyte counts to normal ranges may reduce the competitive advantage of ozanimod on the key safety feature of the lymphocyte recovery profile.

In addition to the effect of ozanimod on circulating lymphocytes, another mode of action has been proposed in the applicability of the drug as a neuroprotective agent; this observation was based on a study in which ozanimod showed the exertion of a beneficial effect on EAE mice even when ALC was normal. ${ }^{24}$ Ozanimod can readily cross the blood-brain barrier and thus potentially exerts

Table I Ozanimod Main Specifics and Chemical Structure

\begin{tabular}{|l|l|}
\hline Drug Names & Ozanimod HCL; Ozanimod Hydrochloride; RPC-I063; Zeposia \\
\hline Class & Sphingosine I-phosphate receptor modulator \\
\hline Route of administration & Oral \\
\hline Phase & $\begin{array}{l}\text { Authorised for CIS, RRMS, and active SPMS by the FDA (March 2020) and for RRMS by the EMA (2020); phase III } \\
\text { trials for inflammatory bowel disease }\end{array}$ \\
\hline Pharmacodynamics & SIPRI and SIPR5 agonist \\
\hline Pivotal Trials & RADIANCE A, RADIANCE B, SUNBEAM \\
\hline Ongoing trials & DAYBREAK, ENLIGHTEN \\
\hline Pharmaceutical company & Bristol-Myers Squibb \\
\hline
\end{tabular}


beneficial effects by directly establishing interactions with brain cells, possibly via S1PR5 signalling. ${ }^{26}$ Studies in mice ${ }^{27,28}$ have shown that ozanimod reduces axonal damage, thereby preserving central nervous system tissue morphology after induced demyelination.

The anti-inflammatory and neuroprotective effects of ozanimod were tested in ex vivo studies in experimental autoimmune encephalomyelitis (EAE) brains. Ozanimod could dampen the EAE glutamatergic synaptic alterations by attenuating the local inflammatory response induced by the leading central nervous system (CNS) cellular actors of EAE synaptopathy (activated microglia and infiltrating $\mathrm{T}$ lymphocytes). ${ }^{29}$

\section{Pharmacokinetics}

Ozanimod is an active molecule that does not require phosphorylation for activation. Its half-life is approximately $20 \mathrm{~h}$; however, owing to its major active metabolite, CC112273, the effective half-life is considerably longer (11 days). ${ }^{30}$ This allows the implementation of a once-daily dosing regimen.

Ozanimod exhibited linear pharmacokinetics with dose-proportional increases in exposure and low-to-moderate inter-subject variability in a double-blind, randomized, placebo-controlled Phase I study. ${ }^{25}$ A steady state was reached within $7 \mathrm{~d}$. It has a high volume of distribution $(5590 \mathrm{~L})$, high binding to plasma proteins (>98\%), and slow absorption (maximum concentrations after 6-8 h) $;^{31}$ this leads to low systemic exposure, thus alleviating the decrease in heart rate observed after the initial dose. ${ }^{25}$

Ozanimod is metabolized via multiple pathways, resulting in the production of numerous circulating metabolites. Two of them, CC112273 and CC1084037, are active metabolites with properties similar to those of ozanimod. Renal clearance plays no significant role in the excretion of ozanimod. ${ }^{25}$

To assess the existence of possible drug-food interactions, a trial involving 24 healthy subjects prescribed with ozanimod along with three different types of the meal was conducted. ${ }^{32}$ Ozanimod blood concentrations were comparable in the fasting, high-fat, and low-fat groups. This data suggests that ozanimod can be ingested regardless of the meal type. However, the consumption of foods containing a considerable amount of tyramine (ie, more than $150 \mathrm{mg}$ ) should be avoided while ingesting ozanimod because of an increased sensitivity to tyramine that may lead to the development of severe hypertension. ${ }^{33}$

\section{Clinical Trials Phase I Trials}

The safety of ozanimod was first tested in two Phase I, randomized, double-blind trials. The data showed a dosedependent reduction in heart rate after the first ozanimod administration; therefore, a dose-escalation protocol was used in all subsequent clinical trials. ${ }^{25}$ These studies also determined that ozanimod did not prolong the QTc interval at therapeutic and supratherapeutic doses and did not raise any new safety concerns. ${ }^{32}$ Furthermore, the effect of ozanimod on circulating leukocyte subsets was evaluated using flow cytometry and epigenetic cell counting in an open label pharmacodynamic study in which MS patients were randomized to oral ozanimod ( 0.5 or $1 \mathrm{mg} / \mathrm{d}$ ) for 12 weeks. Dose-dependent decreases in circulating B- and T-cell counts and differential impacts on naive and memory CD4+ and CD8 $+\mathrm{T}$ cells and CD19+ B cells were observed. $^{34}$

\section{Phase II Trials}

The phase II trial RADIANCE was a 24-week, doubleblind, placebo-controlled clinical trial in RRMS patients, ${ }^{35}$ with the primary endpoint of the cumulative number of total gadolinium-enhancing MRI lesions at weeks 12-24. It showed a reduction in new/enlarging $\mathrm{T} 2$ lesions and GdE lesions on brain MRI scans of patients treated with ozanimod 0.5 or $1 \mathrm{mg} / \mathrm{d}$ versus placebo. Both doses of ozanimod were well tolerated, with nasopharyngitis and headache being the most common TEAEs. After the study conclusion, participants were enrolled in a two-year extension trial, ${ }^{36}$ where ozanimod-treated patients continued the treatment, and the placebo group were randomly switched to ozanimod 0.5 or $1 \mathrm{mg} / \mathrm{d}$. The efficacy results were confirmed with a low number of new $\mathrm{T} 2$ lesions and GdE lesions on brain scans in all groups during the study period. The unadjusted ARR was 0.32 for the group continuing ozanimod $0.5 \mathrm{mg} / \mathrm{d}, 0.30$ for the group switching from placebo to ozanimod $0.5 \mathrm{mg} / \mathrm{d}$, and 0.18 for both groups on ozanimod $1 \mathrm{mg} / \mathrm{d}$ (continuing and switching from placebo). Four patients discontinued treatment because their alanine aminotransferase (ALT) or aspartate aminotransferase (AST) levels increased five-fold compared with their average values. The most common 
TEAEs reported were nasopharyngitis and upper respiratory tract infections.

\section{Phase III Trials}

Two major phase III clinical trials have been conducted: the RADIANCE study and the SUNBEAM study. They used a similar study design, consisting of $7 \mathrm{~d}$ of dose escalation followed by a randomized, double-blind, double-dummy, active-controlled, parallel-group treatment period. The two trials differed principally in the duration of the treatment period.

\section{RADIANCE}

A 24-month, multicentre, double-blind, phase III trial that enrolled 1320 active RRMS patients. ${ }^{36}$

Participants were randomly divided into three groups: daily ozanimod $0.5 \mathrm{mg} / \mathrm{d}$, daily ozanimod $1 \mathrm{mg} / \mathrm{d}$, and weekly intramuscular interferon beta-1a $30 \mu \mathrm{g} / \mathrm{week}$. The treatment period was 24 months. To reduce the possible heart rate decrease effect, an initial 7-d dose escalation was used for ozanimod. The trial demonstrated a lower ARR over 24 months among patients treated with ozanimod (adjusted ARRs were 0.17 with ozanimod $1 \mathrm{mg} / \mathrm{d}$, 0.22 with ozanimod $0.5 \mathrm{mg} / \mathrm{d}$, and 0.28 with interferon beta-1a), with a rate ratio versus interferon beta-1a of 0.79 ( 0.65 to $0.96 ; p=0.0167$ ) for ozanimod $0.5 \mathrm{mg} / \mathrm{d}$ and 0.62 (95\% CI 0.51-0.77; $p<0.0001$ ) for ozanimod $1 \mathrm{mg} / \mathrm{d}$.

The ozanimod-treated groups also showed a fewer new or enlarging T2 lesions over 24 months $(1.84$ for $1 \mathrm{mg} / \mathrm{d}$, 2.09 for $0.5 \mathrm{mg} / \mathrm{d}$, and 3.18 for interferon beta-1a) and a fewer GdE lesions over 24 months ( 0.18 for $1 \mathrm{mg} / \mathrm{d}, 0.20$ for $0.5 \mathrm{mg} / \mathrm{d}$, and 0.37 for interferon beta-1a). Ozanimod showed reduced brain volume loss over 24 months than interferon beta-1a, while the proportion of patients with disability progression at three and six months was not significantly different among the treatment groups. TEAEs were reported by almost $75 \%$ of subjects who received ozanimod and $83 \%$ of subjects who received interferon beta-1a.

Most of the events were mild or moderate. The TEAEs that occurred in more than $5 \%$ of patients treated with ozanimod were nasopharyngitis, pharyngitis, urinary tract infections, and increased ALT and $\gamma$-glutamyl transferase levels. During dose escalation, no clinically meaningful cardiac findings were reported (only asymptomatic and spontaneously resolved bradycardia with $<45 \mathrm{bpm}$ in four patients). No serious opportunistic infections were observed.

\section{SUNBEAM}

A 12-month, multicentre, double-blind, phase III trial that enrolled 1346 active RRMS patients between December 2014 and November $2015^{37}$. As in the RADIANCE trial, subjects were randomly assigned to ozanimod $(0.5 \mathrm{mg} / \mathrm{d})$, ozanimod $(1 \mathrm{mg} / \mathrm{d})$, or interferon beta-1a $(30 \mu \mathrm{g} / \mathrm{week})$. These results are similar to those of RADIANCE. Ozanimod was more effective than interferon beta-1a in lowering the ARR and lowering the number of new T2 lesions and GdE lesions over 12 months. Interestingly, both the RADIANCE and SUNBEAM trial results indicated smaller losses of whole brain volume, cortical grey matter volume, and thalamic volume in patients treated with ozanimod than in those treated with interferon beta-1a. These results have not previously been reported in a phase III study of an MS DMD and suggest a possible role of ozanimod in protecting against structural changes associated with disease progression over time. However, brain atrophy data should be interpreted with caution because of the limitation of the statistical analysis.

A post hoc analyses comparing the effects of ozanimod and interferon beta-1a on cognitive processing speed (CPS) in participants with relapsing MS in SUNBEAM study were recently published. ${ }^{38}$ Ozanimod showed modestly beneficial effects on CPS in RMS participants. In particular, it improved Symbol Digit Modalities Test (SDMT) scores at months 6 and 12. A more significant percentage of ozanimod-treated participants had clinically meaningful improvements in SDMT scores versus interferon beta- $1 \mathrm{a}: 30.0 \%$ vs $22.2 \%$ at month 6 and $35.6 \%$ vs $27.9 \%$ at month 12 . The findings on SDMT have been obtained by conducting a post hoc analysis; thus, a confirmation of this conclusion by performing further studies is mandatory.

TEAEs were reported by $59.8 \%$ of patients on ozani$\bmod 1 \mathrm{mg} / \mathrm{d}, 57.2 \%$ of patients on ozanimod $0.5 \mathrm{mg} / \mathrm{d}$, and $75.5 \%$ of patients on interferon beta-1a. The TEAEs that occurred in more than $5 \%$ of patients treated with ozanimod were nasopharyngitis, upper respiratory tract infections, and headache. No clinically significant bradycardia or second- or third-degree atrioventricular block was observed. No serious opportunistic infections have been reported to date.

The safety results from RADIANCE and SUNBEAM are encouraging; nevertheless, rare events and long-term safety data will be assessed by further ongoing studies and post-marketing surveillance.

A recapitulatory comparison of RADIANCE and SUNBEAM results is shown in Table 2. 


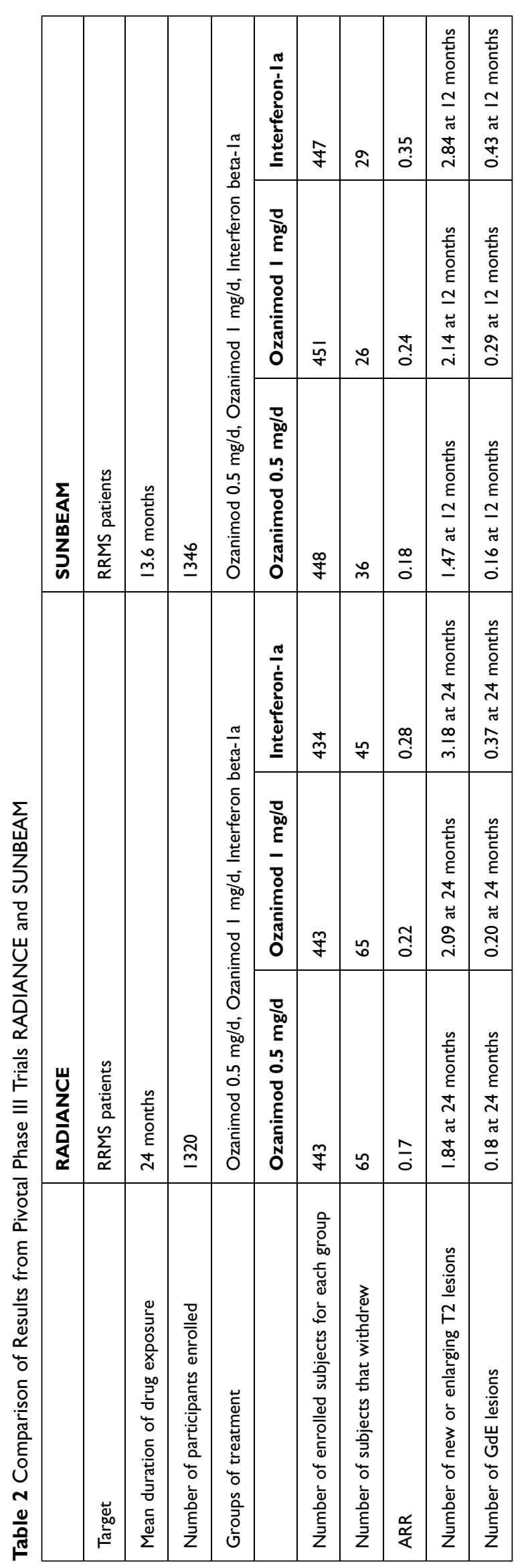




\section{DAYBREAK}

A recently completed open-label phase III trial. The interim results were presented at MS ECTRIMS Meeting in September 2019. ${ }^{39}$ A total of 2495 participants with RRMS who completed a pivotal trial (RADIANCE Phase II, RADIANCE phase III or SUNBEAM) enrolled in DAYBREAK, receiving ozanimod $1 \mathrm{mg} / \mathrm{d}$. This study aimed to evaluate the long-term safety, tolerability, and efficacy of ozanimod. The interim results refer to a population of 2494 subjects exposed for a mean of 19 months. Findings on safety were generally consistent with the phase III studies; the only serious TEAEs that occurred in more than two participants were pyelonephritis (5), uterine leiomyomas (5), and appendicitis (4). TEAEs leading to permanent discontinuation of ozanimod occurred in $1.2 \%$ of the subjects. The overall ARR was 0.124 , with a marked reduction in the ARR of patients who switched from interferon beta-1a (from 0.246 to 0.123 ).

\section{ENLIGHTEN}

An ongoing open-label phase III trial ${ }^{40}$ that evaluates cognitive processing speed changes in RRMS subjects exposed to ozanimod.

\section{A Comparison Between Ozanimod and Other SIPR Modulators}

A recent study ${ }^{41}$ to compare the safety and efficacy outcomes between fingolimod and ozanimod was performed because of the absence of a head-to-head randomized trial. For this purpose, a matching-adjusted indirect comparison was used, using data from pivotal clinical trials of the two drugs. The study showed that the first ozanimod administration was associated with a significantly lower risk of TEAEs than fingolimod administration. In addition, a lower risk of heart rate reduction, atrioventricular blocks, and conduction abnormalities were observed among patients treated with ozanimod. A lower risk of any TEAEs for ozanimod versus fingolimod was also observed after one and two years of treatment. It was also seen that patients treated with ozanimod had a lower risk of TEAEs leading to discontinuation. No statistically significant differences in ARRs and confirmed disability progression were found after three and six months of treatment. Overall, this comparative analysis suggests a favourable safety profile and similar efficacy for ozanimod versus fingolimod. Notably, the gold standard used for comparing two different drugs includes the performance of head-to- head trials. The comparative study reported herein may include biases arising due to the different populations, study design, and pharmacologic eras considered for the execution of the trials.

Another recent study ${ }^{42}$ that might have included the same limitations focused on the comparison of all available S1PR modulators through the conduction of a network meta-analysis (NMA), in which multiple treatments were compared using both direct comparisons of interventions within randomized controlled trials (RCTs) and indirect comparisons across trials based on a common comparator. $^{43}$ An NMA aids better decision-making when head-to-head trials have not been conducted; it suggested that amiselimod (40 $\mathrm{mg}$ ) demonstrated the highest efficacy followed by ponesimod (40 mg). Amiselimod is a selective S1PR1 modulator that exhibited a unique efficacy and safety profile in Phase $2^{44}$ and Expanded Phase 2 trials $;{ }^{45}$ however, its production has been discontinued by Biogen in 2016. In terms of safety, ozanimod (1 mg) was the S1PR modulator demonstrating the lowest risk of serious adverse reaction development that would lead to trial discontinuation. In cases of fingolimod $(1.25 \mathrm{mg})$ and ponesimod $(40 \mathrm{mg})$, higher number of trial dropouts were recorded.

An important concern about fingolimod's safety profile is related to its discontinuation; several studies reported $^{46-50}$ that after discontinuation of the drug, severe clinical and radiological reactivation events were observed in certain patients. There is a lack of agreement regarding the pathological explanation for the so-called "rebound syndrome"; however, it has been postulated that a rapid lymphocyte reconstitution ${ }^{46}$ or a differential subset repopulation ${ }^{51}$ might be responsible for the inflammatory activity.

Thus far, there are no case reports available documenting rebound after ozanimod treatment discontinuation; however, real-world data remain scarce. It is plausible that, considering the similar mechanism of action among S1PR modulators, there may be a risk of severe disease reactivation after ozanimod suspension.

\section{Ozanimod for the Treatment of Other Immune-Mediated Diseases}

Thus far, ozanimod has been approved only for the treatment of relapsing MS; however, other immune-mediated inflammatory diseases may benefit from its use. This is suggested not only by evidence based on similar 
pathophysiology underlying these conditions but also by analyses based on the use of computational predictive models of docking, ${ }^{52}$ however, hypothetical interactions should be confirmed by conducting experimental studies.

A study on the utility of ozanimod in murine models of systemic lupus erythematosus has reported encouraging results. ${ }^{53}$ More advanced research data are available on inflammatory bowel disease (IBD) since two Phase II trials, TOUCHSTONE and STEPSTONE (for ulcerative colitis and Crohn's disease, respectively), have been completed.

The TOUCHSTONE trial ${ }^{54}$ showed modest benefits in moderately treating severely active ulcerative colitis, with clinical remission at eight weeks obtained by $16 \%$ of ozanimod $1 \mathrm{mg} / \mathrm{d}$-treated patients versus $6 \%$ of the placebo group. Ozanimod $0.5 \mathrm{mg}$ did not show a statistically significant benefit. An open-label extension study, ${ }^{55}$ with all patients treated with ozanimod $1 \mathrm{mg} / \mathrm{d}$, showed rapid improvement in Mayo scores for patients who switched from the placebo or ozanimod $0.5 \mathrm{mg} / \mathrm{d}$. Long-term benefit with ozanimod $1 \mathrm{mg}$ daily was displayed in patients with moderate-to-severe ulcerative colitis both on clinical and histologic measures.

The STEPSTONE trial ${ }^{56}$ was an open-label study in which participants received ozanimod $1 \mathrm{mg} / \mathrm{d}$ for 12 weeks. It showed a clinically meaningful improvement in disease activity in patients with moderately to severely active Crohn's disease. A reduction in Crohn's Disease Activity Index (CDAI) was achieved in $66 \%$ of patients, while CDAI remission was achieved by $46 \%$.

Several phase III trials comparing ozanimod to a placebo on ulcerative colitis and Crohn's disease patients are ongoing and recruiting. ${ }^{57}$

Ozanimod is not the only S1PR modulator that is being investigated for applicability in IBD; other novel molecules such as amiselimod, etrasimod, and mocravimod are being studied extensively in clinical trials. ${ }^{58}$

\section{New Horizons for Ozanimod-Based Treatments}

S1PR modulators intervene with a complex mechanism of action, determining circulating lymphocyte regulation and playing an important role in several cellular processes, such as cell survival, migration, and cell-cell communication. The S1P/S1PR signalling pathway is dysregulated in many pathologies, not only in MS and inflammatory bowel diseases. These conditions include several neurologic and non-neurologic diseases, such as degenerative conditions (eg, Alzheimer's disease and amyotrophic lateral sclerosis [ALS]), other immune conditions such as inflammatory bowel disease, psychiatric illnesses (eg, schizophrenia), and evolutive disorders (eg, Rett syndrome). ${ }^{59}$ Several studies that have been conducted for fingolimod, administered for different neurological conditions in vitro and in animal and human models, have shown promising results.${ }^{60-65}$ Overall, the S1PR family appears worthy of further research and may provide significant therapeutic opportunities.

\section{SIPR Modulators and COVID-I 9}

The World Health Organization announced that the outbreak of severe acute respiratory syndrome coronavirus 2 (SARS-CoV-2), causing coronavirus disease 2019 (COVID-19), is a pandemic in March 2020. Many MS patients worldwide have faced this novel coronavirus infection; concerns about the COVID-19 course and concomitant immunosuppressive and immunomodulatory agents have arisen.

On the one hand, immunocompromised patients may show a more severe SARS-CoV-2 infection caused by an inadequate immune response. On the other hand, immunomodulatory and immunosuppressive drugs could mitigate cytokine-release syndrome, resulting in better overall outcomes. ${ }^{66}$

A systematic review focused on evaluating the severity of COVID-19 in MS patients prescribed with different DMTs has been published. ${ }^{67}$ A total of 257 COVID-19 cases, confirmed and suspected, of patients prescribed with fingolimod have been reported. Most patients presented with a relatively mild disease course despite lymphopenia development and showed a complete recovery. Only one death was reported, ${ }^{68}$ tantamounting a death rate of $0.3 \%$. The patient who succumbed to the disease was a 42 -yearold woman presenting with RRMS and an EDSS of 6.0, severe cognitive impairment, and a history of struma treated with radioline; she refused intensive care unit (ICU) admission.

Asymptomatic $^{69}$ SARS-CoV-2 infections and mild ${ }^{70,71}$ and severe ${ }^{72,73}$ COVID-19 case reports in fingolimod-treated MS patients have been published. Bollo ${ }^{70}$ described a favourable outcome in a 34-year-old woman with SARSCoV-2 infection who showed only mild signs of the disease (fever up to 38.5 degree Celsius and pharyngeal pain, without respiratory difficulties); at the time of COVID-19 diagnosis, fingolimod was promptly discontinued. Despite 
a complete recovery, it is noteworthy that the woman showed a limited immunoglobulin response, with negative anti-S1RBD IgG and slightly positive anti-NP IgG presence documented after 35 days. New evidence on the elicitation of immunoglobulin response after the development of COVID-19 in S1PR modulator-treated patients is of interest, since a blunted immune response to SARSCoV-2 antigens may also reduce the efficacy of vaccines.

Another interesting case report published by GomezMayordomo $^{74}$ showed a clinical exacerbation (dyspnoea and lung involvement) of COVID-19 in a 57-year-old man one week after fingolimod discontinuation. Symptoms progressively improved after subjection to steroid therapy.

Only two cases of COVID-19 were reported in MS patients treated with Siponimod ${ }^{75}$ and one case in an MS patient treated with ponesimod. ${ }^{76}$ No deaths were reported.

There is no conclusive evidence regarding the use of S1PR modulators in patients with COVID-19; while their effect on the ALC suggests the exercise of caution, only one case of death out of the 260 cases has been reported thus far; a protective effect on cytokine-releasing syndrome has been postulated thereafter.

\section{Conclusions}

Ozanimod is a new drug in the MS therapeutic panorama belonging to the family of S1PR modulators. Its selectivity for binding to S1PR1 and S1PR5 was hypothesized to be better tolerated than other less selective molecules. Ozanimod was approved in 2020 by the FDA and EMA for relapsing forms of MS in adults ${ }^{77,78}$ after the conclusion of the RADIANCE phase II and III studies and the SUNBEAM phase III study that showed that the drug was effective and well tolerated.

Additional studies are needed to estimate the efficacy of ozanimod in comparison with other therapies. Moreover, it will be mandatory to search for biomarkers to identify the best candidate for S1PR modulator therapies, such as ozanimod, to improve their efficacy and tolerability.

\section{Disclosure}

Nothing to declare related to the present article. MF has nothing to declare. LL serves on scientific advisory boards and received honoraria for speaking from Biogen, Merck, Novartis, Genzyme and Roche. JF serves on scientific advisory boards and received honoraria for speaking from Biogen, Merck, Novartis, Genzyme and Roche. EC serves on scientific advisory boards and received honoraria for speaking and scientific research support from Biogen, Merck, Novartis, Genzyme, Roche and Teva. The authors reported no other potential conflicts of interest for this work.

\section{References}

1. Doshi A, Chataway J. Multiple sclerosis, a treatable disease. Clin Med (Lond). 2016;16(Suppl 6):s53-s59. doi:10.7861/clinmedicine.16-6-s53

2. Oh J, Vidal-Jordana A, Montalban X. Multiple sclerosis: clinical aspects. Curr Opin Neurol. 2018;31(6):752-759. doi:10.1097/ WCO.0000000000000622

3. Obinata H, Hla T. Sphingosine 1-phosphate and inflammation. Int Immunol. 2019;31(9):617-625. doi:10.1093/intimm/dxz037

4. Chaudhry BZ, Cohen JA, Conway DS. Sphingosine 1-Phosphate receptor modulators for the treatment of multiple sclerosis. Neurotherapeutics. 2017;14(4):859-873. doi:10.1007/s13311-017-0565-4

5. Rasche L, Paul F. Ozanimod for the treatment of relapsing remitting multiple sclerosis. Expert Opin Pharmacother. 2018;19(18):20732086. doi:10.1080/14656566.2018.1540592

6. Chiba K, Yanagawa Y, Masubuchi Y, et al. FTY720, a novel immunosuppressant, induces sequestration of circulating mature lymphocytes by acceleration of lymphocyte homing in rats. I. FTY720 selectively decreases the number of circulating mature lymphocytes by acceleration of lymphocyte homing. J Immunol. 1998;160(10):5037-5044.

7. Cohan S, Lucassen E, Smoot K, Brink J, Chen C. Sphingosine-1Phosphate: its pharmacological regulation and the treatment of multiple sclerosis: a review article. Biomedicines. 2020;8(7):227. doi:10.3390/biomedicines8070227

8. Kappos L, Antel J, Comi G, et al. Oral fingolimod (FTY720) for relapsing multiple sclerosis. $N$ Engl J Med. 2006;355(11):1124-1140. doi:10.1056/NEJMoa052643

9. Novgorodov AS, El-Alwani M, Bielawski J, Obeid LM, Gudz TI. Activation of sphingosine-1-phosphate receptor S1P5 inhibits oligodendrocyte progenitor migration. FASEB J. 2007;21(7):1503-1514. doi:10.1096/fj.06-7420com

10. Jaillard C, Harrison S, Stankoff B, et al. Edg8/S1P5: an oligodendroglial receptor with dual function on process retraction and cell survival. $J$ Neurosci. 2005;25(6):1459-1469. doi:10.1523/ JNEUROSCI.4645-04.2005

11. Van Doorn R, Lopes Pinheiro MA, Kooij G, et al. Sphingosine 1phosphate receptor 5 mediates the immune quiescence of the human brain endothelial barrier. $J$ Neuroinflammation. 2012;9:133. doi:10.1186/1742-2094-9-133

12. Urbano M, Guerrero M, Rosen H, Roberts E. Modulators of the Sphingosine 1-phosphate receptor 1. Bioorg Med Chem Lett. 2013;23(23):6377-6389. doi:10.1016/j.bmcl.2013.09.058

13. Gold R, Comi G, Palace J, et al. Assessment of cardiac safety during fingolimod treatment initiation in a real-world relapsing multiple sclerosis population: a Phase 3b, open-label study. J Neurol. 2014;261(2):267-276. doi:10.1007/s00415-013-7115-8

14. Gergely P, Nuesslein-Hildesheim B, Guerini D, et al. The selective sphingosine 1-phosphate receptor modulator BAF312 redirects lymphocyte distribution and has species-specific effects on heart rate. Br J Pharmacol. 2012;167(5):1035-1047. doi:10.1111/j.14765381.2012.02061.x

15. Selmaj K, Li DK, Hartung HP, et al. Siponimod for patients with relapsing-remitting multiple sclerosis (BOLD): an adaptive, doseranging, randomised, phase 2 study [published correction appears in Lancet Neurol. 2013 Sep;12(9):846]. Lancet Neurol. 2013;12(8):756767. doi:10.1016/S1474-4422(13)70102-9

16. Kappos L, Li DK, Stüve O, et al. Safety and Efficacy of Siponimod (BAF312) in patients with relapsing-remitting multiple sclerosis: doseblinded, randomized extension of the phase 2 BOLD Study. JAMA Neurol. 2016;73(9):1089-1098. doi:10.1001/jamaneurol.2016.1451 
17. Kappos L, Bar-Or A, Cree BAC, et al. Siponimod versus placebo in secondary progressive multiple sclerosis (EXPAND): a double-blind, randomised, phase 3 study [published correction appears in Lancet. 2018 Nov 17;392(10160):2170]. Lancet. 2018;391(10127):12631273. doi:10.1016/S0140-6736(18)30475-6

18. Dumitrescu L, Constantinescu CS, Tanasescu R. Siponimod for the treatment of secondary progressive multiple sclerosis. Expert Opin Pharmacother. 2019;20(2):143-150. doi:10.1080/14656566. 2018.1551363

19. U.S. Food and Drug Administration (FDA). FDA approves new oral drug to treat multiple sclerosis; 2021. Available from: https://www. fda.gov/news-events/press-announcements/fda-approves-new-oraldrug-treat-multiple-sclerosis. Accessed March 26, 2019.

20. European Medicines Agency (EMA). Mayzent EPAR; 2021. Available from: https://www.ema.europa.eu/en/medicines/human/ EPAR/mayzent. Accessed November 12, 2019.

21. Olsson T, Boster A, Fernández Ó, et al. Oral ponesimod in relapsingremitting multiple sclerosis: a randomised phase II trial. $J$ Neurol Neurosurg Psychiatry. 2014;85(11):1198-1208. doi:10.1136/jnnp-2013307282

22. Kappos L, Fox RJ, Burcklen M, et al. Ponesimod compared with teriflunomide in patients with relapsing multiple sclerosis in the active-comparator Phase 3 OPTIMUM Study: a Randomized Clinical Trial [published online ahead of print, 2021 Mar 29]. JAMA Neurol. 2021. doi:10.1001/jamaneurol.2021.0405

23. European Medicines Agency (EMA). Ponvory; 2021. Available from: https://www.ema.europa.eu/en/medicines/human/summaries-opinion/ ponvory. Accessed March 25, 2021.

24. Scott FL, Clemons B, Brooks J, et al. Ozanimod (RPC1063) is a potent sphingosine-1-phosphate receptor-1 (S1P1) and receptor-5 (S1P5) agonist with autoimmune disease-modifying activity. $\mathrm{Br} \mathrm{J}$ Pharmacol. 2016;173(11):1778-1792. doi:10.1111/bph.13476

25. Tran JQ, Hartung JP, Peach RJ, et al. Results from the first-in-human study with ozanimod, a novel, selective sphingosine-1-Phosphate receptor modulator. J Clin Pharmacol. 2017;57(8):988-996. doi: $10.1002 /$ jcph. 887

26. ECTRIMS Online Library. Taylor Meadows K Ozanimod (RPC1063) is potentially neuroprotective through direct CNS effects. 10/27/17; 200838; P118310/27/17; 200838; P1183. Available from: https://online library.ectrims-congress.eu/ectrims/2017/ACTRIMS-ECTRIMS2017/ 200838/kristen.r.taylor.meadows.ozanimod.28rpc106329.is.potentially. neuroprotective.html. Accessed April 23, 2021.

27. Taylor Meadows K, Scott F, Villescaz C, et al. Ozanimod (RPC1063) reduces the plasma biomarker neurofilament light chain in preclinical rodent models of multiple sclerosis. ECTRIMS Online Library; 2021. Available from: https://onlinelibrary.ectrims-congress.eu/ectrims/2017/ ACTRIMS-ECTRIMS2017/199629/kristen.r.taylor.meadows.ozanimod. (rpc1063).reduces.the.plasma.biomarker.html. Accessed April 23, 2021.

28. Taylor Meadows K Ozanimod (RPC1063) is potentially neuroprotective through direct CNS effects. ECTRIMS online library; 2021. Available from: https:/onlinelibrary.ectrims-congress.eu/ectrims/2017/ACTRIMSECTRIMS2017/200838/kristen.r.taylor.meadows.ozanimod. 28rpc106329.is.potentially.neuroprotective.html. Accessed April 23, 2021.

29. Musella A, Gentile A, Guadalupi L, et al. Central modulation of selective sphingosine-1-Phosphate receptor 1 ameliorates experimental multiple sclerosis. Cells. 2020;9(5):1290. doi:10.3390/ cells9051290

30. Cree BAC, Mares J, Hartung HP. Current therapeutic landscape in multiple sclerosis: an evolving treatment paradigm [published correction appears in Curr Opin Neurol. 2019 Oct;32(5):782]. Curr Opin Neurol. 2019;32(3):365-377. doi:10.1097/WCO.0000000000000700

31. Celgene Corporation. Zeposia (ozanimod) capsules: US prescription information 2020; 2021. Available from: https://www.accessdata.fda. gov/drugsatfda_docs/label/2020/209899s000lbl.pdf. Accessed April 23, 2021.
32. Tran JQ, Hartung JP, Tompkins CA, Frohna PA. Effects of high- and low-fat meals on the pharmacokinetics of ozanimod, a novel sphingosine-1-Phosphate receptor modulator. Clin Pharmacol Drug Dev. 2018;7(6):634-640. doi:10.1002/cpdd.409

33. ZEPOSIA (Ozanimod) (Package Insert). Celgene Corporation: summit, NJ, USA; 2021. Available from: https://www.accessdata.fda.gov/drug satfda_docs/label/2020/209899s000lbl.pdf. Accessed April 23, 2021.

34. Harris S, Tran JQ, Southworth H, Spencer CM, Cree BAC, Zamvil SS. Effect of the sphingosine-1-phosphate receptor modulator ozanimod on leukocyte subtypes in relapsing MS. Neurol Neuroimmunol Neuroinflamm. 2020;7(5):e839. doi:10.1212/NXI.0000000000000839

35. Cohen JA, Arnold DL, Comi G, et al. Safety and efficacy of the selective sphingosine 1-phosphate receptor modulator ozanimod in relapsing multiple sclerosis (RADIANCE): a randomised, placebocontrolled, phase 2 trial. Lancet Neurol. 2016;15(4):373-381. doi:10.1016/S1474-4422(16)00018-1

36. Cohen JA, Comi G, Selmaj KW, et al. Safety and efficacy of ozanimod versus interferon beta-1a in relapsing multiple sclerosis (RADIANCE): a multicentre, randomised, 24-month, phase 3 trial. Lancet Neurol. 2019;18(11):1021-1033. doi:10.1016/S1474-4422 (19)30238-8

37. Comi G, Kappos L, Selmaj KW, et al. Safety and efficacy of ozanimod versus interferon beta-1a in relapsing multiple sclerosis (SUNBEAM): a multicentre, randomised, minimum 12-month, phase 3 trial. Lancet Neurol. 2019;18(11):1009-1020. doi:10.1016/ S1474-4422(19)30239-X

38. DeLuca J, Schippling S, Montalban X, et al. Effect of ozanimod on symbol digit modalities test performance in relapsing MS [published online ahead of print, 2020 Dec 10]. Mult Scler Relat Disord. 2020;48:102673. doi:10.1016/j.msard.2020.102673

39. ECTRIMS Online Library. Steinman L. 09/12/19; 278233; P1031. Long-term safety and efficacy of ozanimod in relapsing multiple sclerosis: results from the DAYBREAK Open-Label Extension Study; 2021. Available from: https://onlinelibrary.ectrims-congress. eu/ectrims/2019/stockholm/278233/lawrence.steinman.long-term. safety.and.efficacy.of.ozanimod.in.relapsing.html. Accessed April 23, 2021.

40. The ENLIGHTEN study; 2021. Available from: https://clinicaltrials. gov/ct2/show/NCT03261453. Accessed April 23, 2021.

41. Swallow E, Patterson-Lomba O, Yin L, et al. Comparative safety and efficacy of ozanimod versus fingolimod for relapsing multiple sclerosis. J Comp Eff Res. 2020;9(4):275-285. doi:10.2217/cer-2019-0169

42. Tong J, Zou Q, Chen Y, et al. Efficacy and acceptability of the S1P receptor in the treatment of multiple sclerosis: a meta-analysis [published online ahead of print, 2021 Feb 1] [published correction appears in Neurol Sci. 2021 Mar 19;:]. Neurol Sci. 2021. doi:10.1007/s10072-021-05049-w

43. $\mathrm{Lu} \mathrm{G}$, Ades AE. Combination of direct and indirect evidence in mixed treatment comparisons. Stat Med. 2004;23(20):3105-3124. doi:10.1002/sim.1875

44. Kappos L, Arnold DL, Bar-Or A, et al. Safety and efficacy of amiselimod in relapsing multiple sclerosis (MOMENTUM): a randomised, double-blind, placebo-controlled phase 2 trial. Lancet Neurol. 2016;15(11):1148-1159. doi:10.1016/S1474-4422(16)30192-2

45. Kappos L, Arnold DL, Bar-Or A, et al. Two-year results from a phase 2 extension study of oral amiselimod in relapsing multiple sclerosis. Mult Scler. 2018;24(12):1605-1616. doi:10.1177/1352458517728343

46. Hakiki B, Portaccio E, Giannini M, Razzolini L, Pastò L, Amato MP. Withdrawal of fingolimod treatment for relapsing-remitting multiple sclerosis: report of six cases. Mult Scler. 2012;18(11):1636-1639. doi: $10.1177 / 1352458512454773$

47. Berger B, Baumgartner A, Rauer S, et al. Severe disease reactivation in four patients with relapsing-remitting multiple sclerosis after fingolimod cessation. $J$ Neuroimmunol. 2015;282:118-122. doi:10.1016/j.jneuroim.2015.03.022 
48. Ghezzi A, Rocca MA, Baroncini D, et al. Disease reac- tivation after fingolimod discontinuation in two multiple sclerosis patients. $J$ Neurol. 2013;260:327-329. doi:10.1007/s00415-012-6744-7

49. Hatcher SE, Waubant E, Nourbakhsh B, Crabtree-Hartman E, Graves JS. Rebound syndrome in patients with multiple sclerosis after cessation of fingolimod treatment. JAMA Neurol. 2016;73(7):790-794. doi:10.1001/jamaneurol.2016.0826

50. Frau J, Sormani MP, Signori A, et al. Clinical activity after fingolimod cessation: disease reactivation or rebound? Eur J Neurol. 2018;25(10):1270-1275. doi:10.1111/ene.13694

51. Song ZY, Yamasaki R, Kawano Y, et al. Peripheral blood T cell dynamics predict relapse in multiple sclerosis patients on fingolimod. PLoS One. 2015;10(4):e0124923. doi:10.1371/journal.pone.0124923

52. Al-Zaqri N, Pooventhiran T, Jagadeeswara Rao D, et al. Structure, conformational dynamics, quantum mechanical studies and potential biological activity analysis of multiple sclerosis medicine ozanimod. J Mol Struc. 2021;1227:e129685. doi:10.1016/j.molstruc.2020.129685

53. Taylor Meadows KR, Steinberg MW, Clemons B, et al. Ozanimod (RPC1063), a selective S1PR1 and S1PR5 modulator, reduces chronic inflammation and alleviates kidney pathology in murine systemic lupus erythematosus. PLoS One. 2018;13(4):e0193236. doi:10.1371/journal.pone.0193236

54. Sandborn WJ, Feagan BG, Wolf DC, et al. Ozanimod induction and maintenance treatment for ulcerative colitis. $N$ Engl J Med. 2016;374 (18):1754-1762. doi:10.1056/NEJMoa1513248

55. Sandborn WJ, Feagan BG, Hanauer S. et al. Long-term efficacy and safety of ozanimod in moderate-to-severe ulcerative colitis: results from the open-label extension of the randomized, Phase 2 Touchstone Study [published online ahead of print, 2021 Jan 13]. J Crohns Colitis;2021. jjab012. doi:10.1093/ecco-jcc/jjab012

56. Feagan BG, Sandborn WJ, Danese S, et al. Ozanimod induction therapy for patients with moderate to severe Crohn's disease: a single-arm, phase 2, prospective observer-blinded endpoint study. Lancet Gastroenterol Hepatol. 2020;5(9):819-828. doi:10.1016/ S2468-1253(20)30188-6

57. Lamb YN. Ozanimod: first Approval. Drugs. 2020;80(8):841-848. doi:10.1007/s40265-020-01319-7

58. Pérez-Jeldres T, Tyler CJ, Boyer JD, et al. Targeting cytokine signaling and lymphocyte traffic via small molecules in inflammatory bowel disease: JAK Inhibitors and S1PR Agonists. Front Pharmacol. 2019;10:212. doi:10.3389/fphar.2019.00212

59. O'Sullivan S, Dev KK. Sphingosine-1-phosphate receptor therapies: advances in clinical trials for CNS-related diseases. Neuropharmacology. 2017;113(Pt $\quad$ B):597-607. doi:10.1016/j. neuropharm.2016.11.006

60. Potenza RL, De Simone R, Armida M, et al. Fingolimod: a diseasemodifier drug in a mouse model of amyotrophic lateral sclerosis. Neurotherapeutics. 2016;13(4):918-927. doi:10.1007/s13311-0160462-2

61. Deogracias R, Yazdani M, Dekkers MP, et al. Fingolimod, a sphingosine-1 phosphate receptor modulator, increases BDNF levels and improves symptoms of a mouse model of Rett syndrome. Proc Natl Acad Sci U S A. 2012;109(35):14230-14235. doi:10.1073/ pnas. 1206093109

62. Li W, Xu H, Testai FD, Mechanism of action and clinical potential of fingolimod for the treatment of stroke. Front Neurol. 2016;7:139. doi:10.3389/fneur.2016.00139

63. Estrada-Bernal A, Palanichamy K, Ray Chaudhury A, Van Brocklyn JR. Induction of brain tumor stem cell apoptosis by FTY720: a potential therapeutic agent for glioblastoma. Neuro Oncol. 2012;14 (4):405-415. doi:10.1093/neuonc/nos005
64. Di Pardo A, Amico E, Favellato M, et al. FTY720 (fingolimod) is a neuroprotective and disease-modifying agent in cellular and mouse models of Huntington disease. Hum Mol Genet. 2014;23(9):22512265. doi:10.1093/hmg/ddt615

65. Gao F, Liu Y, Li X, Wang Y, Wei D, Jiang W. Fingolimod (FTY720) inhibits neuroinflammation and attenuates spontaneous convulsions in lithium-pilocarpine induced status epilepticus in rat model. Pharmacol Biochem Behav. 2012;103(2):187-196. doi:10.1016/j. pbb.2012.08.025

66. Zheng C, Kar I, Chen CK, et al. Multiple sclerosis disease-modifying therapy and the COVID-19 Pandemic: implications on the Risk of Infection and Future Vaccination. CNS Drugs. 2020;34(9):879-896. doi:10.1007/s40263-020-00756-y

67. Sharifian-Dorche M, Sahraian MA, Fadda G, et al. COVID-19 and disease-modifying therapies in patients with demyelinating diseases of the central nervous system: a systematic review [published online ahead of print, 2021 Jan 29]. Mult Scler Relat Disord. 2021;50:102800. doi:10.1016/j.msard.2021.102800

68. Loonstra FC, Hoitsma E, van Kempen ZL, Killestein J, Mostert JP. COVID-19 in multiple sclerosis: the Dutch experience. Mult Scler. 2020;26(10):1256-1260. doi:10.1177/1352458520942198

69. Mallucci G, Zito A, Fabbro BD, Bergamaschi R. Asymptomatic SARS-CoV-2 infection in two patients with multiple sclerosis treated with fingolimod. Mult Scler Relat Disord. 2020;45:102414. doi:10.1016/j.msard.2020.102414

70. Bollo L, Guerra T, Bavaro DF, et al. Seroconversion and indolent course of COVID-19 in patients with multiple sclerosis treated with fingolimod and teriflunomide. J Neurol Sci. 2020;416:117011. doi:10.1016/j.jns.2020.117011

71. Bowen JD, Brink J, Brown TR, et al. COVID-19 in MS: initial observations from the Pacific Northwest. Neurol Neuroimmunol Neuroinflamm. 2020;7(5):e783. doi:10.1212/NXI.0000000000000783

72. Barzegar M, Mirmosayyeb O, Nehzat N, et al. COVID-19 infection in a patient with multiple sclerosis treated with fingolimod. Neurol Neuroimmunol Neuroinflamm. 2020;7(4):e753. doi:10.1212/ NXI.0000000000000753

73. Foerch C, Friedauer L, Bauer B, Wolf T, Adam EH. Severe COVID19 infection in a patient with multiple sclerosis treated with fingolimod. Mult Scler Relat Disord. 2020;42:102180. doi:10.1016/j. msard.2020.102180

74. Gomez-Mayordomo V, Montero-Escribano P, Matías-Guiu JA, González-García N, Porta-Etessam J, Matías-Guiu J. Clinical exacerbation of SARS-CoV2 infection after fingolimod withdrawal. $J$ Med Virol. 2020;93:546-549. doi:10.1002/jmv.26279

75. Parrotta E, Kister I, Charvet L, et al. COVID-19 outcomes in MS: observational study of early experience from NYU Multiple Sclerosis Comprehensive Care Center. Neurol Neuroimmunol Neuroinflamm. 2020;7(5):e835. doi:10.1212/NXI.0000000000000835

76. Sormani MP, De Rossi N, Schiavetti I, et al. Disease-modifying therapies and coronavirus disease 2019 severity in multiple sclerosis. Ann Neurol. 2021;89:780-789. doi:10.1002/ana.26028

77. U.S. Food and Drug Administration (FDA). Drug Trials Snapshots: ZEPOSIA; 2021. Available from: https://www.fda.gov/drugs/develop ment-approval-process-drugs/drug-trials-snapshots-zeposia. Accessed April 23, 2021.

78. European Medicines Agency (EMA). Zeposia; 2021. Available from: https://www.ema.europa.eu/en/medicines/human/EPAR/zeposia. Accessed April 23, 2021. 


\section{Publish your work in this journal}

Drug Design, Development and Therapy is an international, peerreviewed open-access journal that spans the spectrum of drug design and development through to clinical applications. Clinical outcomes, patient safety, and programs for the development and effective, safe, and sustained use of medicines are a feature of the journal, which has also been accepted for indexing on PubMed Central. The manuscript management system is completely online and includes a very quick and fair peer-review system, which is all easy to use. Visit http://www. dovepress.com/testimonials.php to read real quotes from published authors. 